\title{
Impact of lanadelumab in hereditary angioedema: a case series of 12 patients in Canada
}

\author{
Aled laboni ${ }^{*}\left(\mathbb{D}\right.$, Amin Kanani ${ }^{2}$, Gina Lacuesta ${ }^{3}$, Christine Song ${ }^{4}$, Manstein Kan ${ }^{5}$ and Stephen D. Betschel ${ }^{6}$
}

\begin{abstract}
Background: Hereditary angioedema (HAE) is a rare autosomal dominant disease resulting in recurring episodes of swelling, leading to considerable patient morbidity and mortality. Lanadelumab is a plasma kallikrein inhibitor that is approved as 1st line therapy in Canada for long term prophylaxis of HAE attacks.

Objective: To describe our clinical findings from a case series of adult patients with HAE type 1/2 who have been initiated on lanadelumab.

Methods: A chart review of HAE type 1/2 patients at three academic centers in Canada was undertaken with demographic and clinical data extracted. Patients were included if they had been receiving lanadelumab for at least 6 months. Patients with other causes of angioedema were excluded.

Results: 12 patients meeting enrollment criteria were identified. Compared to pre-lanadelumab, patients had mean reductions of $72 \%$ and $62 \%$ in attack rate and treated attack rate respectively. 3 patients reported complete remission from attacks after starting lanadelumab. Most patients had significant improvements in HAE impact on social outings.

Conclusion: Our case series findings support the 2019 International/Canadian HAE guideline that lanadelumab is an effective therapy for long term prophylaxis. In our patient population, initiation of lanadelumab improved disease control, minimized the burden of treatment and improved HAE impact on social outings.
\end{abstract}

Keywords: Lanadelumab, Hereditary angioedema, HAE, Swelling

\section{Background}

Hereditary angioedema (HAE) is a rare genetic disease resulting in recurring episodes of painful swelling of the subcutaneous and submucosal tissue [1]. Attacks can affect many areas of the body. Most frequently involved are the extremities, gastrointestinal tract, genitals, face and upper airway. Swelling of the larynx can occur with potentially fatal consequences due to asphyxiation [2]. HAE patients with recurrent attacks have functional

\footnotetext{
*Correspondence: aled.iaboni@unityhealth.to

${ }^{1}$ Allergy/Immunology Fellow, Department of Internal Medicine, Division of Clinical Immunology and Allergy, University of Toronto, St. Michael's Hospital, Toronto, ON, Canada

Full list of author information is available at the end of the article
}

impairment, reduced quality of life (QoL) and increased mortality $[3,5]$. The prevalence of HAE is approximately 1:50,000 individuals [3]. The condition is equally prevalent among sexes. Although quite variable, the mean age of onset for clinical symptoms is 11 years of age [4].

HAE is divided into 3 categories based on $\mathrm{C} 1$ esterase inhibitor enzyme (C1-INH) level and function. Patients who have quantitatively low levels of C1-INH are deemed to have type $1 \mathrm{HAE}$ (HAE-1) and make up $85 \%$ of all cases. Those with a normal C1-INH antigenic level but dysfunctional enzyme activity are classified as type 2 HAE (HAE-2) and make up approximately $15 \%$ of cases [6]. HAE $1 / 2$ result from mutations in the SERPING1 original author(s) and the source, provide a link to the Creative Commons licence, and indicate if changes were made. The images or other third party material in this article are included in the article's Creative Commons licence, unless indicated otherwise in a credit line to the material. If material is not included in the article's Creative Commons licence and your intended use is not permitted by statutory regulation or exceeds the permitted use, you will need to obtain permission directly from the copyright holder. To view a copy of this licence, visit http://creativecommons.org/licenses/by/4.0/. The Creative Commons Public Domain Dedication waiver (http://creativeco mmons.org/publicdomain/zero/1.0/) applies to the data made available in this article, unless otherwise stated in a credit line to the data. 
gene and are inherited in a predominantly autosomal dominant fashion [7]. A third category of HAE has normal C1-INH (HAE nC1-INH) and clinically presents similarly to HAE $1 / 2$. HAE nC1-INH is exceedingly rare and the true prevalence is not known. Identified causes of HAE nC1-INH include genetic mutations in factor XII, angiopoietin 1, plasminogen, kininogen 1 and myoferlin [8]. In this case series, we will be addressing only patients with $\mathrm{HAE} 1 / 2$.

In HAE, attacks of swelling are caused by overproduction of bradykinin that acts as a potent vasodilator. C1-INH inhibits various proteins along the bradykinin pathway including factor XIIa and plasma kallikrein. In HAE $1 / 2, \mathrm{C} 1$-INH deficiency results in unopposed plasma kallikrein activity and overproduction of bradykinin. This overproduction causes vascular permeability and characteristic attacks of swelling [9].

Therapeutic options for HAE involve treatment of acute attacks, short term prophylaxis (STP), and long term prophylaxis (LTP). In Canada, licensed options available for treatment of acute attacks include icatibant, a bradykinin B2 receptor antagonist, and plasma-derived C1-INH (pdC1-INH). STP refers to the prophylactic treatment of HAE patients to reduce the risk of an attack during a high risk period such as dental surgery or upper airway manipulation. The treatment of choice for STP is intravenous (IV) pdC1-INH within an hour prior to the procedure. LTP is the use of ongoing, scheduled therapy to reduce attack frequency/severity and improve QoL in patients who are unable to meet their treatment goals with on-demand therapy alone. First line options for LTP in Canada include subcutaneous (SC) pdC1-INH and lanadelumab. Available second line options for LTP in some patients include attenuated androgens such as danazol and tranexamic acid (TXA), an antifibrinolytic [3].

Lanadelumab (Takhzyro ${ }^{\mathrm{TM}}$ ) is a fully humanized monoclonal antibody and is a highly potent and specific plasma kallikrein inhibitor, thereby reducing generation of bradykinin. It has a half-life of $\sim 2$ weeks and is given subcutaneously as a $300 \mathrm{mg}$ injection once every 2 weeks, though this interval can be extended to 4 weeks if attacks are controlled for $>6$ months $[10,11]$. The HELP study was a Phase 3 , randomized clinical trial (RCT) of Lanadelumab vs. Placebo involving 125 patients with HAE $1 / 2$. The study showed that compared to placebo, lanadelumab significantly reduced attack frequency/ severity and improved QoL over a 26 week treatment period [12]. The HELP study open-label extension (OLE) has since shown sustained reduction in attack frequency and a good drug safety profile [13]. Lanadelumab is now approved in Canada for use as LTP for HAE $1 / 2$ in patients $\geq 12$ years of age. Any patient $\geq 12$ years of age with HAE $1 / 2$ in Canada can be started on lanadeleumab for LTP. The decision to start lanadelumab is made jointly by the patient and treating physician and is based upon many factors including efficacy of on-demand therapy, frequency/severity of attacks and disease impact on QoL. It is not necessary to have failed other LTP agents before initiating lanadelumab [3].

To the best of our knowledge, there have been no reports outlining the clinical efficacy of lanadelumab in Canadian HAE patients. Herein, we describe our findings from a case series of 12 adult patients with HAE 1/2 who have been initiated on LTP with lanadelumab.

\section{Patients and methods}

A retrospective chart review was conducted from three academic hospitals in Canada examining patients with HAE $1 / 2$ who receive LTP with lanadelumab. The following inclusion criteria were used: Patients must have HAE $1 / 2$ and be receiving lanadelumab for at least 6 months at the time of study enrollment. The diagnosis of HAE $1 / 2$ must be established for enrolled patients based on a history of characteristic attacks of swelling and either low C1-INH level (Type 1) or low C1-INH function (Type 2). Patients with other etiologies of angioedema (histaminergic, acquired, or HAE nC1-INH) were excluded. While patient 10 (comorbid multiple myeloma) has not had acquired angioedema actively ruled out, her diagnosis of HAE 1 was established 18 years prior the onset of myeloma features. Patient 12 (comorbid T1DM) has not had acquired angioedema actively ruled out as his father also had HAE and died of upper airway angioedema, suggesting genetic inheritance. Enrollment for the study was completed March, 2021. All patients enrolled in the study were started on lanadelumab through compassionate initiation of the drug. Data was extracted from chart review and includes basic patient demographics and clinical information both before and after transition to lanadelumab.

A total of 12 patients were identified for this case series. From Toronto Ontario, Vancouver British Columbia, and Halifax Nova Scotia there were 7, 3, and 2 patients enrolled respectively. Demographic data are reported in Table 1. The mean age of enrolled patients is 43 years. The average age of HAE symptom onset was 18 years old. Excluding Patient 3, who was diagnosed prior to symptom onset due to an affected family member, the average latency from HAE symptom onset to diagnosis was 8 years. Most patients enrolled in the case series had few comorbidities. Patients 3 and 5 have a history of systemic lupus erythematosus and ulcerative colitis respectively (both quiescent), while Patient 10 is currently on maintenance therapy for Multiple Myeloma. 
Table 1 Data of 11 HAE patients gathered from chart review including demographics, comorbidities, HAE type and time of diagnosis

\begin{tabular}{|c|c|c|c|c|c|c|c|}
\hline Case & Age & Sex & HAE Type & Comorbidities & Other medications & $\begin{array}{l}\text { Age symptom } \\
\text { onset (years) }\end{array}$ & $\begin{array}{l}\text { Age HAE } \\
\text { diagnosis } \\
\text { (years) }\end{array}$ \\
\hline 1 & 25 & M & 1 & Nil & $\mathrm{Nil}$ & 18 & 18 \\
\hline 2 & 24 & M & 1 & Nil & Nil & 1 & 4 \\
\hline 3 & 42 & $\mathrm{~F}$ & 1 & SLE (quiescent) & $\mathrm{Nil}$ & 10 & 23 \\
\hline 4 & 38 & $\mathrm{~F}$ & 2 & Nil & Nil & 11 & 2 \\
\hline 5 & 36 & $\mathrm{~F}$ & 1 & Ulcerative Colitis & $\mathrm{Nil}$ & 4 & 12 \\
\hline 6 & 30 & $\mathrm{~F}$ & 1 & GERD, Chronic Pain & Methadone & 23 & 25 \\
\hline 7 & 59 & $\mathrm{~F}$ & 1 & $\mathrm{Nil}$ & $\mathrm{Nil}$ & 26 & 59 \\
\hline 8 & 34 & $\mathrm{~F}$ & 1 & Depression & Citalopram & 11 & 11 \\
\hline 9 & 50 & $\mathrm{~F}$ & 1 & $\mathrm{Nil}$ & $\mathrm{Nil}$ & 47 & 48 \\
\hline 10 & 74 & $\mathrm{~F}$ & 1 & Multiple Myeloma, HTN & Revlimid, Telmisartan & Unknown & 55 \\
\hline 11 & 44 & $\mathrm{~F}$ & 1 & Raynaud's, GERD, Cervical Stenosis & Esomeprazole & 15 & 33 \\
\hline 12 & 65 & M & 1 & T1DM, Primary Adrenal Insufficiency & $\begin{array}{l}\text { Insulin } \\
\text { Pantoprazole }\end{array}$ & 37 & 37 \\
\hline
\end{tabular}

GERD gastroesophageal reflux disease, SLE systemic lupus erythematosus, HTN hypertension, T1DM type 1 diabetes

All clinical data were extracted from patient clinical encounter notes. Pre-lanadelumab clinical data were gathered from all notes in the year prior to initiating lanadelumab. Post-lanadelumab clinical data were gathered from the most recent 12 months of clinical notes. HAE impact on social outings (ISO) was gathered and coded by the treating MD on a scale of 1 to $5(1=$ no impact, $3=$ some impact, $5=\mathrm{a}$ lot of impact) by $\mathrm{MD}$ interpretation of patient clinical encounter notes.

\section{Results}

After a chart review of patient clinical encounter notes from pre- and post-lanadelumab, HAE disease manifestations and therapy history were extracted (Table 2). The type and prevalence HAE attacks experienced by patients were extremity swelling (9/12), abdominal pain (10/12), laryngeal swelling (7/12), lip swelling (3/12) and headaches (1/12). The attacks stated to be most debilitating were abdominal (8/12), laryngeal (4/12), and extremity swelling (1/11). Prior to initiating lanadelumab, most patients had received LTP with either IV or SC pdC1-INH (10/12). Patients 7 and 9 had never used LTP prior to initiating lanadelumab. All patients were started on lanadelumab between April 2019 and October 2020, which was made available for compassionate use, and remained on this therapy without interruption. The lanadelumab dosage used for $11 / 12$ patients was $300 \mathrm{mg}$ SC once every 2 weeks. Patient 4 had been switched from every 2 weeks to every 4 weeks in September, 2020 due to absence of attacks in the preceding 6 months. Acute treatment used by patients to treat breakthrough attacks was most commonly icatibant $30 \mathrm{mg}$ SC.
Data from case series patients was extracted including HAE attack frequency, number of treated attacks, missed work days and HAE ISO (Table 3). The median number of attacks/year and treated attacks/year pre-lanadelumab was $11(\min =3, \max =300)$ and $6(\min =0, \max =50)$ respectively. The median HAE ISO pre-lanadelumab was $3(\min =2, \max =5)$. There were 3 patients who had experienced missed work days as a result of HAE in the year prior to initiating lanadelumab.

The median number of attacks/year and treated attacks/year post-lanadelumab was $3 \quad(\min =0$, $\max =100)$ and $2(\min =0, \max =40)$ respectively. Patients 4, 9 and 12 were completely free of attacks since initiating lanadelumab. The mean reduction in attack rate and treated attack rate post-lanadelumab transition was $72 \%$ and $62 \%$ respectively (Fig. 1). The median HAE ISO post-lanadelumab was $1(\min =1, \max =3)$ with an average reduction of 1.8 compared to pre-lanadelumaab (Fig. 2). Post-lanadelumab, 8 out of 12 patients reported no social outing impact from their HAE. Only Patient 6 remained off work due to their HAE.

\section{Discussion and conclusions}

Our case series demonstrates that LTP with lanadelumab resulted in considerable reductions in HAE attack rates in all patients enrolled in the study. Three of these patients had complete remission of $\mathrm{HAE}$ attacks. Most patients reported less impact of their HAE on social outings following initiation of therapy. It is notable that these improvements were seen in 10/12 patients who had been previously using LTP with pdC1INH. The 2019 International/Canadian HAE guideline has recommended lanadelumab and SC pdC1-INH 
Table 2 Clinical characteristics and HAE therapy history of case series patients

\begin{tabular}{|c|c|c|c|c|c|c|c|}
\hline Case & Type of attacks & $\begin{array}{l}\text { Most debilitating } \\
\text { attacks }\end{array}$ & Pre-lana LTP & $\begin{array}{l}\text { Pre-lana acute } \\
\text { treatment }\end{array}$ & $\begin{array}{l}\text { Lana transition } \\
\text { date }\end{array}$ & Lana dosage & $\begin{array}{l}\text { Post-lana acute } \\
\text { treatment }\end{array}$ \\
\hline 1 & $\begin{array}{l}\text { Extremity swelling } \\
\text { Abdominal pain } \\
\text { Laryngeal swelling }\end{array}$ & Laryngeal swelling & $\begin{array}{l}\text { C1-INH 2000U IV } \\
\text { q3-4d }\end{array}$ & Icatibant 30 mg SC & January 2020 & $300 \mathrm{mg}$ SC q2w & Icatibant 30 mg SC \\
\hline 2 & $\begin{array}{l}\text { Abdominal pain } \\
\text { Lip swelling }\end{array}$ & Abdominal pain & $\begin{array}{l}\text { C1-INH } 3000 \text { U IV } \\
\text { q3d }\end{array}$ & C1-INH 3000U IV & July 2019 & $300 \mathrm{mg}$ SC q2w & C1-INH 3000U IV \\
\hline 3 & $\begin{array}{l}\text { Extremity swelling } \\
\text { Laryngeal swelling }\end{array}$ & Laryngeal swelling & $\begin{array}{l}\text { C1-INH 1500U IV } \\
\text { q5d }\end{array}$ & Icatibant 30 mg SC & January 2020 & $300 \mathrm{mg}$ SC q2w & Icatibant 30 mg SC \\
\hline 4 & $\begin{array}{l}\text { Extremity swelling } \\
\text { Abdominal pain }\end{array}$ & Abdominal pain & $\begin{array}{l}\text { C1-INH 1500U IV } \\
\text { q3-4d }\end{array}$ & Icatibant 30 mg SC & October 2019 & $300 \mathrm{mg}$ SC q4w & Icatibant 30 mg SC \\
\hline 5 & $\begin{array}{l}\text { Extremity swelling } \\
\text { Abdominal pain } \\
\text { Lip swelling } \\
\text { Headaches }\end{array}$ & Abdominal pain & $\begin{array}{l}\text { C1-INH 1500U IV } \\
\text { q3-4d }\end{array}$ & C1-INH 3000 U IV & April 2020 & $300 \mathrm{mg}$ SC q2w & C1-INH 1500U IV \\
\hline 6 & $\begin{array}{l}\text { Abdominal pain } \\
\text { Extremity swelling } \\
\text { Laryngeal swelling }\end{array}$ & Abdominal pain & $\begin{array}{l}\text { C1-INH 1500U IV } \\
\text { q3d }\end{array}$ & Icatibant 30 mg SC & April 2019 & $300 \mathrm{mg}$ SC q2w & Icatibant 30 mg SC \\
\hline 7 & $\begin{array}{l}\text { Abdominal pain } \\
\text { Extremity swelling } \\
\text { Laryngeal swelling }\end{array}$ & Abdominal pain & None & None & October 2020 & $300 \mathrm{mg}$ SC q2w & Icatibant 30 mg SC \\
\hline 8 & $\begin{array}{l}\text { Abdominal pain } \\
\text { Extremity swelling }\end{array}$ & Abdominal pain & $\begin{array}{l}\text { C1-INH } 4500 \text { U SC } \\
\text { q3-4d }\end{array}$ & Icatibant 30 mg SC & February 2020 & $300 \mathrm{mg}$ SC q2w & Icatibant 30 mg SC \\
\hline 9 & Extremity swelling & Extremity swelling & None & Prednisone $^{a}$ & February 2020 & $300 \mathrm{mg}$ SC q2w & Icatibant 30 mg SC \\
\hline 10 & $\begin{array}{l}\text { Abdominal pain } \\
\text { Extremity swelling } \\
\text { Laryngeal swelling }\end{array}$ & Abdominal attacks & $\begin{array}{l}\text { Danazol C1-INH } \\
\quad 4500 \text { U SC q3-4d }\end{array}$ & C1-INH IV & May 2019 & $300 \mathrm{mg}$ SC q2w & C1-INH IV \\
\hline 11 & $\begin{array}{l}\text { Lip swelling } \\
\text { Abdominal pain } \\
\text { Laryngeal swelling }\end{array}$ & Laryngeal swelling & $\begin{array}{l}\text { TXA C1-INH } 1500 U \\
\text { IV q2-3d }\end{array}$ & $\begin{array}{l}\text { Icatibant or C1-INH } \\
\text { IV }\end{array}$ & January 2020 & $300 \mathrm{mg}$ SC q2w & $\begin{array}{l}\text { Icatibant or } \mathrm{C} 1-\mathrm{INH} \\
\text { IV }\end{array}$ \\
\hline 12 & $\begin{array}{l}\text { Abdominal pain } \\
\text { Laryngeal swelling }\end{array}$ & $\begin{array}{r}\text { Abdominal, } \\
\text { Laryngeal }\end{array}$ & $\begin{array}{l}\text { Danazol C1-INH } \\
\text { 2000U IV q3-4d }\end{array}$ & $\begin{array}{l}\text { Icatibant or } \mathrm{C} 1-\mathrm{INH} \\
\text { IV }\end{array}$ & October 2020 & $300 \mathrm{mg}$ SC q2w & Icatibant \\
\hline
\end{tabular}

All data was extracted from patient chart review

LTP long-term prophylaxis, C1-INH plasma-derived C1 esterase inhibitor enzyme, TXA tranexamic acid

${ }^{\text {a }}$ Prednisone was given to this patient as acute treatment prior to HAE diagnosis and was ineffective at treating attacks

as $1^{\text {st }}$ line options for HAE-1/2 LTP (Consensus level of evidence) [3]. Our case series findings support this recommendation and show that lanadelumab is effective in the real-world Canadian HAE-1/2 patient population.

While not directly comparable, our study showed less efficacy of lanadelumab than the HELP study. Results from the HELP study show that HAE patients on lanadelumab $300 \mathrm{mg}$ every 2 weeks had a mean attack rate reduction of $92.5 \%$ compared to the run-in period (0.26 vs. 3.5 attacks/month). $48.1 \%$ of patients on this dose were attack free [12]. This difference in results could be explained by the extensive use of pdC1-INH in our patient population. Only $40.7 \%$ of patients in the HELP study (lanadelumab $300 \mathrm{mg}$ every 2 weeks arm) were on prophylaxis with pdC1-INH prior to enrolling in the study compared to $83 \%$ in our study. The increased use of LTP with pdC1-INH in our patient population prior to initiating lanadelumab likely resulted in better baseline disease control and reduced the magnitude of attack rate reduction after transitioning to lanadelumab.

Both IV and SC pdC1-INH used for LTP have been shown to reduce attack frequency, duration and severity in HAE $1 / 2$ as seen in the CHANGE and COMPACT studies $[14,15]$. To date, there have been no head-tohead trials comparing lanadelumab to these agents. A recent indirect treatment comparison study was done to compare LTP efficacy in the HELP trial (lanadelumab $300 \mathrm{mg}$ SC every 2 or 4 weeks) and the CHANGE study (IV pdC1-INH 1000U every 3-4 days). The study showed a statistically significant reduction in HAE attack rate by $73 \%$ and $46 \%$ with lanadelumab $q 2 \mathrm{w}$ and $\mathrm{q} 4 \mathrm{w}$ compared to IV pdC1-INH [16]. In line with findings from this indirect treatment comparison, 9/12 of our case series patients previously used IV C1-INH prior to switching to lanadelumab and all had significant reductions in attack rates after this switch. Our cases and this indirect treatment comparison provide evidence 
Table 3 Case series data of the number of total/laryngeal HAE attacks in the year prior to initiating lanadelumab compared to the year following initiation of lanadelumab

\begin{tabular}{|c|c|c|c|c|c|c|c|c|c|c|}
\hline \multirow[t]{2}{*}{ Case } & \multicolumn{5}{|c|}{ Pre-lanadelumab transition } & \multicolumn{5}{|c|}{ Post-lanadelumab transition } \\
\hline & Attacks/year & $\begin{array}{l}\text { Laryngeal } \\
\text { attacks/year }\end{array}$ & $\begin{array}{l}\text { Treated } \\
\text { attacks/ } \\
\text { year }\end{array}$ & $\begin{array}{l}\text { Missed work } \\
\text { days/year }\end{array}$ & ISO & Attacks /year & $\begin{array}{l}\text { Laryngeal } \\
\text { attacks/year }\end{array}$ & $\begin{array}{l}\text { Treated } \\
\text { attacks/ } \\
\text { year }\end{array}$ & $\begin{array}{l}\text { Missed work } \\
\text { days/year }\end{array}$ & ISO \\
\hline 1 & 10 & 4 & 6 & 6 & 2 & 2 & 0 & 2 & 0 & 1 \\
\hline 2 & 12 & 0 & 6 & 20 & 2 & 4 & 0 & 0 & 0 & 1 \\
\hline 3 & 8 & 2 & 8 & 0 & 4 & 2 & 1 & 2 & 0 & 2 \\
\hline 4 & 6 & 0 & 6 & 0 & 3 & 0 & 0 & 0 & 0 & 1 \\
\hline 5 & 50 & 0 & 25 & 0 & 5 & 20 & 0 & 20 & 0 & 2 \\
\hline 6 & 300 & 50 & 50 & Offa & 5 & 100 & 40 & 40 & Off & 1 \\
\hline 7 & 15 & 0 & 0 & Off & 4 & 12 & 0 & 0 & $\mathrm{Off}^{\mathrm{b}}$ & 1 \\
\hline 8 & 6 & 0 & 6 & 0 & 3 & 2 & 0 & 2 & 0 & 2 \\
\hline 9 & 10 & 0 & 6 & 0 & 2 & 0 & 0 & 0 & 0 & 1 \\
\hline 10 & 30 & 0 & 12 & Retired & 3 & 6 & 0 & 6 & Retired & 1 \\
\hline 11 & 12 & 0 & 12 & $\mathrm{Off}^{3}$ & 2 & 6 & 0 & 6 & Offe & 1 \\
\hline 12 & 3 & 0 & 3 & Off ${ }^{4}$ & 3 & 0 & 0 & 0 & Off & 3 \\
\hline
\end{tabular}

Treated attacks are the number of times an acute attack was treated with breakthrough therapy. ISO was determined from MD review of patient clinical encounter notes pre- and post-lanadelumab. ISO, impact on social outings ( $1=$ no impact, $3=$ some, $5=$ a lot $)$

${ }^{a}$ This patient is unable to work due to the frequency of her attacks

${ }^{b}$ This patient is a homemaker and is not employed

'This patient is off work due to a prior motor vehicle accident in 1999 causing cervical stenosis and progressive myelopathy

${ }^{d}$ This patient is off work due to chronic comorbid medical conditions (T1DM, Adrenal Insufficiency)

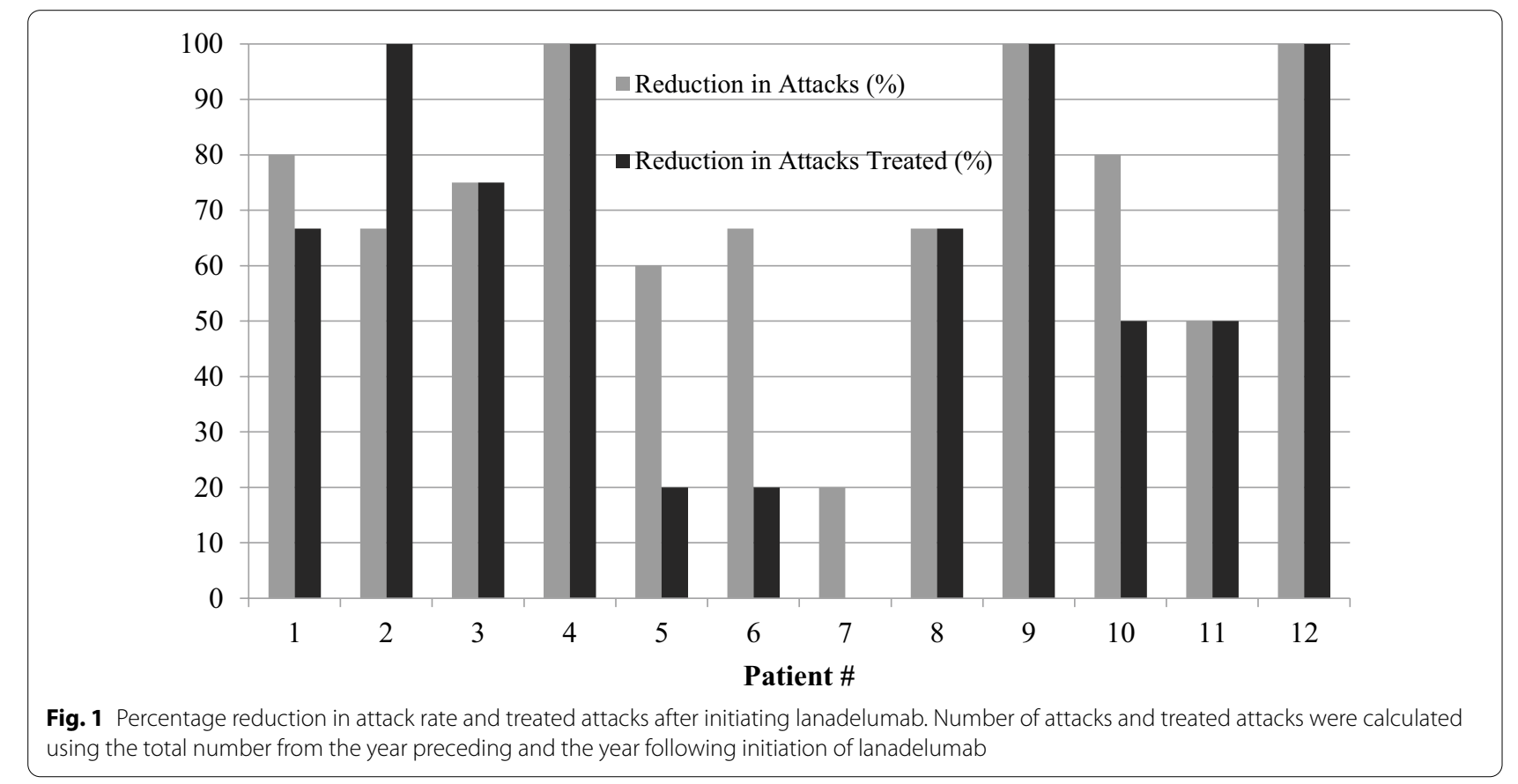

that lanadelumab results in potentially improved efficacy when used as LTP compared to IV pdC1-INH, though a head to head trial would be required to confirm this. An ITC has not yet been performed between the HELP and COMPACT studies but would be useful to compare efficacy between lanadelumab and SC pdC1-INH.

In addition to the suspected advantage in efficacy over IV pdC1-INH, lanadelumab is also easier to 


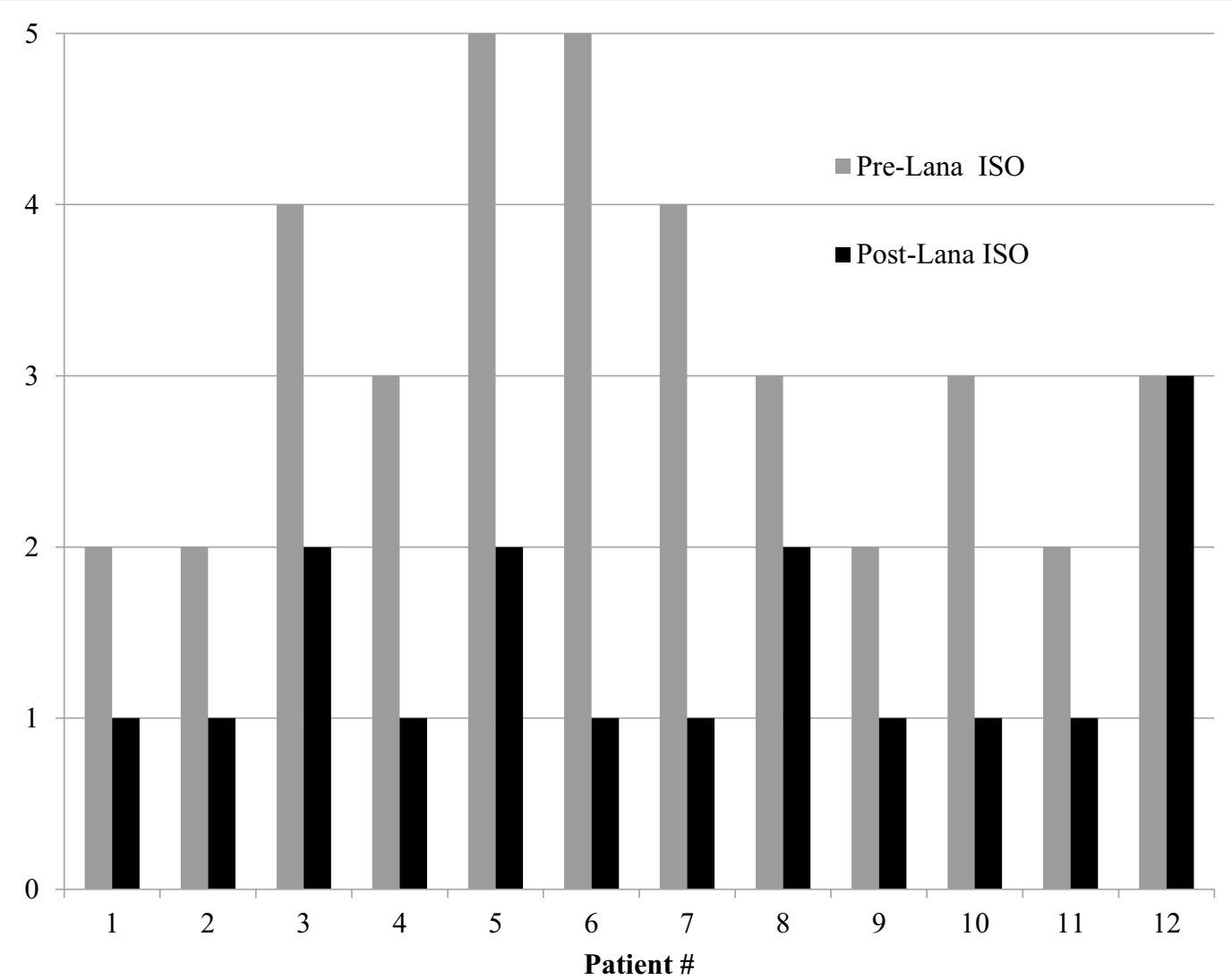

Fig. 2 HAE impact on social outings (ISO) prior to and following initiation of lanadelumab. ISO was coded on a scale of 1 to $5(1=$ none, $3=$ some, $5=$ a lot). Pre-lanadelumab ISO was based on MD records prior to lanadelumab initiation. Post-lanadelumab ISO was based on MD records from the most recent patient encounter since starting lanadelumab

self-administer through the SC route which is likely to improve compliance. An interim analysis from the HELP study OLE showed that $70.9 \%$ of patients preferred the SC route of administration compared to IV pdC1-INH [17]. Compared to SC pdC1-INH that is dosed every 3-4 days, lanadelumab is more convenient to administer as it is dosed every $2-4$ weeks.

Overall, our case series supports the existing 2019 International/Canadian HAE guideline that lanadelumab is effective when used as LTP in reducing patient attack rates, ISO and missed work days in the Canadian HAE $1 / 2$ population. Our findings suggest that patients with HAE refractory to LTP with IV pdC1-INH, and possibly SC pdC1-INH, could consider switching to lanadelumab to attain better disease control and minimize the burden of treatment. A headto-head comparison between these agents would be beneficial to support this recommendation. A possible limitation of our study is that all patients received lanadelumab through compassionate access, which could lead to them perceiving and reporting better disease control while on the drug. Future areas that remain to be explored include the role lanadelumab as LTP in acquired angioedema and HAE nC1-INH.

\section{Abbreviations \\ HAE: Hereditary angioedema; QoL: Quality of life; C1-INH: C1 esterase inhibitor enzyme; HAE-1: Type 1 hereditary angioedema; HAE-2: Type 2 hereditary angioedema; HAE nC1-INH: Hereditary angioedema with normal C1 esterase inhibitor enzyme; STP: Short term prophylaxis; LTP: Long term prophylaxis; pdC1-INH: Plasma-derived C1-INH; IV: Intravenous; SC: Subcutaneous; TXA: Tranexamic acid; RCT: Randomized clinical trial; SLE: Systemic lupus erythematosus; GERD: Gastroesophageal reflux disease; HTN: Hypertension; ISO: Impact on social outings; ITC: Indirect treatment comparison; OLE: Open label extension.}

\section{Acknowledgements}

Nil.

\section{Authors' contributions}

All authors contributed to the study conception. All authors completed chart review and extracted data for the purposes of this submission. The first draft and manuscript was written by Al and all authors commented on previous versions of the manuscript. All authors read and approved the final manuscript.

\section{Funding}

No applicable funding sources for this research. 


\section{Availability of data and materials}

All data collected for the purposes of this study comply with field standards. The datasets used and/or analyzed during the current study are available from the corresponding author on reasonable request.

\section{Declarations}

\section{Ethics approval and consent to participate}

This is a case series based on chart review. Upon review of The Unity Health Research Ethics board protocols and guidelines, it was determined that ethics approval was not required for this type of submission.

\section{Consent for publication}

The case subjects freely gave informed consent to participate in this research.

\section{Competing interests}

Al: This author declares that they have no competing interests.SB: Speaker and/or advisor for CSL Behring, Shire/Takeda. Funding of research CSL Behring.AK: Speaker and/or advisor for CSL Behring, Shire/Takeda. Funding of research from Takeda.GL: Speaker and/or advisor for CSL Behring, Shire/ Takeda. Industry sponsored research with Takeda.CS: This author declares that they have no competing interests.MK: This author declares that they have no competing interests.

\section{Author details}

${ }^{1}$ Allergy/Immunology Fellow, Department of Internal Medicine, Division of Clinical Immunology and Allergy, University of Toronto, St. Michael's Hospital, Toronto, ON, Canada. ${ }^{2}$ Division of Allergy and Immunology, Department of Medicine, St. Paul's Hospital, University of British Columbia, Vancouver, BC, Canada. ${ }^{3}$ Department of Medicine, Nova Scotia Health Authority, Dalhousie University, Halifax, NS, Canada. ${ }^{4}$ Department of Medicine, Division of Allergy and Immunology, St. Michael's Hospital, University of Toronto, Toronto, ON, Canada. ${ }^{5}$ Division of Allergy and Immunology, Department of Medicine, University of British Columbia, Vancouver, BC, Canada. ${ }^{6}$ Medicine and Departmental Division Director for Clinical Immunology and Allergy, Division of Clinical Immunology and Allergy, University of Toronto, St. Michael's Hospital, Toronto, ON, Canada.

Received: 29 March 2021 Accepted: 13 July 2021

Published online: 23 July 2021

\section{References}

1. Nussberger J, Cugno M, Amstutz C, Cicardi M, Pellacani A, Agostoni A. Plasma bradykinin in angio-oedema. Lancet. 1998;351:1693-7.

2. Bork K, Hardt J, Witzke G. Fatal laryngeal attacks and mortality in hereditary angioedema due to C1-INH deficiency. J Allergy Clin Immunol. 2012;130(3):692-7.

3. Betschel S, Badiou J, Binkley K, Borici-Mazi R, Hébert J, Kanani A, et al. The international/Canadian hereditary angioedema guideline. Allergy Asthma Clin Immunol. 2019;15(72):1-29.
4. Bork K, Meng G, Staubach P, Hardt J. Hereditary angioedema: new findings concerning symptoms, affected organs, and course. Am J Med. 2006:119:267-74

5. Lee E, Hsieh J, Borici-Mazi R, Caballero T, Kanani A, Lacuesta G, et al. Quality of life in patients with hereditary angioedema in Canada. Ann Allergy Asthma Immunol. 2021;S1081-1206(21):00002-8. https://doi.org/ 10.1016/j.anai.2021.01.002.

6. Busse P, Christiansen S. Hereditary angioedema. N Engl J Med. 2020;382:1136-48.

7. Ponard D, Gaboriaud C, Charignon D, Ghannam A, Wagenaar-Bos I, Roem D, et al. SERPING1 mutation update: mutation spectrum and C1 Inhibitor phenotypes. Hum Mutat. 2020;41(1):38-57.

8. Bork K, Machnig T, Wulff K, Witzke G, Prusty S, Hardt J. Clinical features of genetically characterized types of hereditary angioedema with normal C1 inhibitor: a systematic review of qualitative evidence. Orphanet J Rare Dis. 2020;15(1):289.

9. Zuraw B. The pathophysiology of hereditary angioedema. World Allergy Organ J. 2010;3:S25-8.

10. Banerji A, Busse P, Shennak M, Lumry W, Davis-Lorton M, Wedner H, et al. Inhibiting plasma kallikrein for hereditary angioedema prophylaxis. N Engl J Med. 2017;376:717-28.

11. Takhzyro Product Monograph Sept 2020. https://www.takeda.com/ 4ab39a/siteassets/en-ca/home/what-we-do/our-medicines/productmonographs/takhzyro/takhzyro-pm-en.pdf. Accessed 11 Mar 2021.

12. Banerji A, Riedl M, Bernstein J, Cicardi M, Longhurst $H$, Zuraw B, et al. Effect of lanadelumab compared with placebo on prevention of hereditary angioedema attacks: a randomized clinical trial. JAMA. 2018;320:2108-21.

13. Johnston $D$, Banerji A, Nurse C, Lu P, Aygören-Pürsün E, Kiani-Alikhan $\mathrm{S}$, Soteres $\mathrm{D}$, et al. Long-term safety of lanadelumab in hereditary angioedema (HAE): interim results from the HELP OLE Study. Ann Allergy Asthma Immunol. 2019;123:S30.

14. Zuraw B, Baker J, Cartwright W, Patel P, Tanner D, Redhead J, et al. Nanofiltered $\mathrm{C} 1$ inhibitor concentrate for treatment of hereditary angioedema. N Engl J Med. 2010;363:513-22.

15. Longhurst H, Cicardi M, Craig T, Bork K, Grattan C, Baker J, et al. Prevention of hereditary angioedema attacks with a subcutaneous $\mathrm{C} 1$ inhibitor. N Engl J Med. 2017;376(12):1131-40.

16. Mendivil J, Malmenäs M, Haeussler K, Hunger M, Jain G, Devercelli G. Indirect comparison of lanadelumab and intravenous $\mathrm{C} 1-\mathrm{NNH}$ using data from the HELP and CHANGE studies: Bayesian and frequentist analyses. Drugs R\&D. 2021;21:113-21

17. Lumry W, Busse P, Lu P, Jain G, Nurse C, Maurer M. Subcutaneous selfadministration of lanadelumab for prophylactic treatment in patients with hereditary angioedema (HAE). Ann Allergy Asthma Immunol. 2018;121(5):S57. https://doi.org/10.1016/j.anai.2018.09.186.

\section{Publisher's Note}

Springer Nature remains neutral with regard to jurisdictional claims in published maps and institutional affiliations.

Ready to submit your research? Choose BMC and benefit from:

- fast, convenient online submission

- thorough peer review by experienced researchers in your field

- rapid publication on acceptance

- support for research data, including large and complex data types

- gold Open Access which fosters wider collaboration and increased citations

- maximum visibility for your research: over 100M website views per year

At BMC, research is always in progress.

Learn more biomedcentral.com/submissions 